\title{
Grußwort zur djb-Veranstaltung „Aktionärinnen fordern Gleichberechtigung“
}

\section{Präsentation der djb-Studie 2010 am 1. Dezember 2010 in der Bertelsmann Repräsentanz, Berlin}

\begin{abstract}
Liz Mohn
Geschäftsführerin der Bertelsmann Verwaltungsgesellschaft, Sprecherin der Familie Bertelsmann, Mitglied des Aufsichtsrates und des Personalausschusses der Bertelsmann AG, Stellv. Vorsitzende des Vorstandes und des Kuratoriums der Bertelsmann Stiftung
\end{abstract}

Liebe Frau Wagner,

sehr geehrte Frau Staatssekretärin,

sehr geehrter Herr Staatssekretär,

liebe Frau Peschel-Gutzeit,

meine Damen und Herren,

liebe Gäste,

willkommen im Hause Bertelsmann in Berlin!

Seit ich denken kann, haben mich Themen wie „Frauenförderung“, „Balance von Beruf und Familie“ oder „Frauen in Führungspositionen“ begleitet.

Schließlich stehe ich seit Jahrzehnten im Beruf und habe drei Kinder - spreche also aus eigener Erfahrung.

Das Haus Bertelsmann repräsentiere ich in der fünften Generation des Familienunternehmens, meine Kinder bereits in der sechsten.

Wir sind uns der Verantwortung für die Menschen, die bei Bertelsmann arbeiten, sehr bewusst.

Dies galt für unsere Familie zu Zeiten des Biedermeier, dies gilt heute und wird auch morgen Bestand haben. Denn Bertelsmann und seine Mitarbeiter sind unser Leben.

Gemeinsam werden wir weiterhin dafür sorgen, dass die Werte lebendig gehalten und weitergetragen werden, für die mein Mann Reinhard Mohn stand und für die ich heute als Sprecherin meiner Familie stehe:

Partnerschaft, Unternehmergeist, Kreativität und gesellschaftliche Verantwortung.

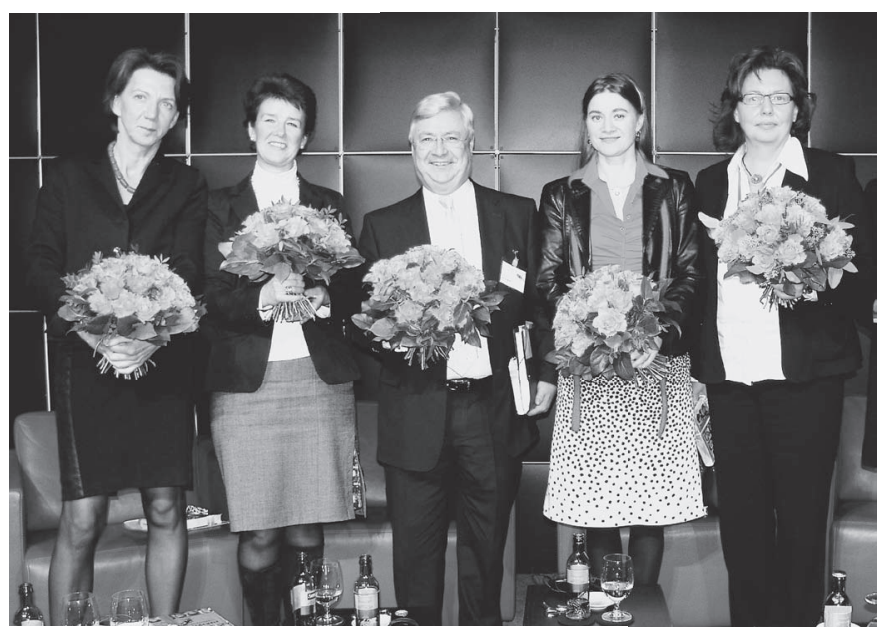

Das soziale und gesellschaftliche Engagement nach innen wie nach außen zieht sich wie ein roter Faden kontinuierlich durch 175 Jahre unserer Familiengeschichte. Lassen Sie mich drei Beispiele nennen:

- Schon 1887 richtete Bertelsmann seine erste betriebliche Alters- und Invalidenkasse ein.

- Seit 1970 beteiligen wir unsere Mitarbeiter am Unternehmenserfolg - wer hat seinerzeit schon an dieses Modell geglaubt?

- Und sieben Jahre später wurde die operative Bertelsmann Stiftung gegründet, die sich seit nunmehr 33 Jahren im Sinne des Gemeinwohls engagiert.

Kein anderes Medienhaus verfügt über so eine lange Geschichte. Kein anderes ist so international: In mehr als 50 Ländern beschäftigen wir heute über hunderttausend Mitarbeiter.

Damit Sie meine Rolle bei Bertelsmann einordnen können, möchte ich Ihnen kurz meine Funktionen erläutern:

Zum einen bin ich als Geschäftsführerin der Bertelsmann Verwaltungsgesellschaft aktiv.

Hier werden alle wichtigen strategischen Entscheidungen getroffen. Dem Gremium gehören sechs Personen an: drei Mitglieder der Familie und drei externe Unternehmer.

Als Sprecherin der Familie widme ich mich bei Bertelsmann insbesondere der Weiterentwicklung der von meinem Mann Reinhard Mohn aufgebauten Unternehmenskultur.

Seit 40 Jahren arbeite ich in der Bertelsmann AG. Dort bin ich als Mitglied des Aufsichtsrates und des Personalausschusses tätig.

In der Bertelsmann Stiftung arbeite ich außerdem als Stellvertretende Vorsitzende des Vorstandes und des Kuratoriums.

Wir alle wissen, dass durch die weltweiten Herausforderungen der Globalisierung überall der Wettbewerbs-Druck steigt. Vor allem die Anforderungen an die Führung wachsen ständig.

Umso wichtiger ist es, exzellente Führungskräfte heranzubilden. Denn: Eine gute Führung ist und bleibt die Voraussetzung für den Erfolg.

Bei Bertelsmann haben wir über Jahrzehnte die Erfahrung gemacht:

Delegation der Verantwortung und eine partnerschaftliche Unternehmenskultur, die auch die Bedürfnisse der Mitarbeiter berücksichtigt, ist nicht der einfachste, aber der erfolgreichste Weg. Ihre Gestaltung ist Chefsache!

Auf dem Podium: Vera Gäde-Butzlaff, Dr. Birgit Grundmann, Prof. Dr. h.c. Klaus-Peter Müller, Anke Domscheit-Berg, Sabine Kauper (v.I.n.r.).

Foto: @ Sharon Adler, Aviva-Berlin 
Und sie stellt hohe Ansprüche an die Führungskraft. Oft sind schwierige Entscheidungen zu fällen. Das bedeutet viele schlaflose Nächte!

Bereits seit 1977 fragen wir die Mitarbeiter direkt nach ihren Erfahrungen. 2010 erfolgte die dritte weltweite Mitarbeiterbefragung: das Votum von 70.000 Menschen aus 400 Firmen in 47 Ländern. 85,1 Prozent aller Mitarbeiter beteiligten sich!

Dieses beeindruckende Engagement zeigt, wie wichtig das Instrument für eine gelebte Partnerschaft im Unternehmen ist.

Meine Damen und Herren,

es ist schon erstaunlich: Auch 2010 nennen laut Umfrage der Bertelsmann Stiftung noch fast 90 Prozent der Deutschen „Familie und Kinder“ als Hauptgrund dafür, dass Frauen in Beruf und Gesellschaft benachteiligt werden.

Offensichtlich wandelt sich das Rollenverständnis in unserem Land nur sehr langsam.

Zwei Drittel aller Deutschen - und immerhin auch die Hälfte der befragten Männer! - sind überzeugt, dass Frauen und Männer nach wie vor keine gleichen Chancen im Beruf haben.

Dass viele junge Menschen unter Dreißig dieses anders sehen, finde ich sehr erfreulich.

Wenig überrascht hat mich, dass Frauen vor allem die Doppelbelastung von Familie und Beruf als Karriere-Hindernis ansehen.

In der Bertelsmann Stiftung setze ich mich daher seit Jahren für Projekte zur Vereinbarkeit von Familie und Beruf ein - anfangs gemeinsam mit Ministerin Renate Schmidt, dann mit Frau von der Leyen, jetzt mit Ministerin Kristina Schröder und Frau Peschel-Gutzeit.

Wir fördern Initiativen zu Führung, Unternehmenskultur und gesellschaftlicher Verantwortung von Unternehmen.

Was also ist zu tun in unserem Land?

Neue Konzepte zur frühkindlichen Betreuung sind der richtige Weg.

Wir brauchen eine ausreichende Zahl von Kitaplätzen (für ein Drittel aller Kinder unter drei Jahren) und auch mehr Betriebskindergärten.

Auch die Ganztagsbetreuung in Schulen könnte einen wichtigen Beitrag leisten.

In den skandinavischen Ländern zum Beispiel ist es viel einfacher, Familie und Beruf in Einklang zu bringen.

Das zahlt sich dann letztlich - wie in Skandinavien - auch in höheren Geburtenraten aus:

Die Frauen im Norden Europas bringen im Laufe ihres Lebens 37 Prozent mehr Kinder zur Welt. (in Deutschland sind es exakt 1,38 „Kinder pro Frau“; in Skandinavien: 1,89).
Sie wissen, meine Damen und Herren: bei uns in Deutschland sind die Frauen in vielen Feldern auf dem Weg nach vorn. Die Frauen-Erwerbsquote liegt bereits heute bei 66,8 Prozent.

Sowohl im Abitur-Notendurchschnitt als auch in zahlreichen Studien-Abschlüssen - sogar in den Naturwissenschaften - sind Frauen heute auf dem Vormarsch, haben zum Teil sogar ihre männlichen Kollegen überholt.

Damit es künftig mehr als derzeit zehn Prozent der Frauen in die Führungsetagen schaffen, brauchen wir vor allem eine Gemeinschafts-Aktion von Wirtschaft, Politik, Unternehmen und Verbänden.

\section{Zwischen jetzt - 1. Stufe - und Quote liegen noch eine} Menge möglicher Schritte, die man gehen muss.

\section{Dr. Birgit Grundmann}

Wir können es uns nicht länger leisten, das „Gold“ in den Köpfen der vielen exzellent ausgebildeten Frauen brach liegen zu lassen!

Ich habe selbst erfahren, wie es ist, als Frau eine Führungsposition einzunehmen.

Daher habe ich im vergangenen Jahr eine Akademie für weibliche Führungskräfte ins Leben gerufen.

Es hat sich nämlich gezeigt, dass Frauen oft das nötige Wissen um eine Strategie fehlt, wie sie aktiv ihre Karriere in der Firma voranbringen können:

\section{Ich bin nicht jemand, der Frauen verhindert.} Die Anzahl meiner männlichen Freunde ist sicher nicht gestiegen.

\section{Prof. Dr. Klaus-Peter Müller}

Die meisten sehen Chancen anders als ihre männlichen Kollegen und warten oft zu lange, bis sie ihre Möglichkeiten wahrnehmen.

Sie sollten mehr Selbstvertrauen zeigen und ihr Durchhaltevermögen beweisen.

Und Betriebe und Unternehmen würden gut daran tun, Frauen in ihrer Organisation ganz gezielt auf Führungspositionen anzusprechen und zu qualifizieren.

Ich bin überzeugt: Berufstätigkeit ist immer auch ein wichtiger Teil zur Persönlichkeitsentwicklung und Sinnerfüllung - für Männer und für Frauen. 
Und was, meine Damen und Herren, können nun Frauen selbst tun, um ihre Talente und Stärken in Wirtschaft und Gesellschaft besser zur Geltung zu bringen?

Ich denke, Frauen müssen lernen, Chancen nicht nur zu sehen, sondern auch für sich zu nutzen bzw. zu beanspruchen.

Dazu gehört, dass sie ihre wertvollen Kompetenzen hervorheben und ins Unternehmen einbringen - und dass sie aktiv an ihrer Persönlichkeit arbeiten.

\section{Stichwort: Statussymbole}

Manchmal werde ich gefragt: Wie sieht denn der richtige Weg in der Führung aus? Ich sage: diesen einen richtigen Weg gibt es nicht! Jede Frau sollte sich auf ihren eigenen Weg konzentrieren.

Meine Empfehlung an alle Damen:

- Blicken Sie öfter mal über den eigenen „Tellerrand“ Ihres Arbeitsplatzes hinaus!
- Suchen Sie das Gespräch mit anderen und tauschen sich über Ihre Gedanken aus!

- Knüpfen Sie Netzwerke - schließen Sie Freundschaften seien Sie offen für Dialoge - suchen Sie sich Ratgeber und hinterfragen Sie sich selbst!

Jeder Mensch sollte sich auch immer wieder bewusst machen: Man trägt nicht nur Verantwortung für andere - man muss auch Verantwortung für sich selbst übernehmen.

Aus meiner 40-jährigen Berufserfahrung habe ich gelernt, was es bedeutet, Freiraum und Gestaltungsmöglichkeiten zu nutzen:

Ich habe auf der ganzen Welt viele unglaublich interessante Menschen kennengelernt und immer wieder erlebt, was Sinnerfüllung heißt.

Und meine wichtigste Erfahrung war immer wieder: Letztendlich gewinnt die Menschlichkeit.

Vielen Dank.

\section{Vorstellung der Studie „Aktionärinnen fordern Gleichberechtigung“}

\section{Dezember 2010, Bertelsmann Repräsentanz, Berlin}

\section{Prof. Dr. Marlene Schmidt}

Vorsitzende der Kommission Arbeits-, Gleichstellungs- und Wirtschaftsrecht des djb; Rechtsanwältin, Frankfurt a.M.

Meine sehr verehrten Damen und Herren,

in dieser 129-seitigen Veröffentlichung finden sich neben der Auswertung unseres Projekts „Aktionärinnen fordern Gleichberechtigung “ eine Fülle von Überlegungen, wie eine wirksame Erhöhung des Frauenanteils in Führungspositionen - insbesondere Aufsichtsratspositionen - deutscher Unternehmen bewerkstelligt werden kann. Ich möchte Ihnen im Folgenden zunächst die Auswertung der Hauptversammlungsteilnahmen vorstellen. Anschließend greife ich beispielhaft einige der sonstigen Beiträge heraus, um deutlich zu machen, welche kreativen Ideen die Mitglieder des Deutschen Juristinnenbunds entwickelt haben in der Hoffnung, dass möglichst viele von ihnen aufgegriffen und umgesetzt werden.

\section{Das Projekt „Aktionärinnen fordern Gleichberechtigung“}

Ziel des Projekts war es nicht in erster Linie, empirische Daten zu erheben, sondern das Thema Frauen in Führungspositionen sollte durch die Fragen in den Hauptversammlungen über die Anteilseigner und die Presse eine größere Öffentlichkeit erreichen und es sollte eine Sensibilisierung für dieses Thema stattfinden. Bei den Hauptversammlungen wurden Fragen zu Diversity und konkreten Maßnahmen zur Förderung von Frauen in Führungspositionen gestellt. Weiterhin wurde nach Frauenförderprogrammen und Veröffentlichungen von frau- enspezifischen Daten und Kennziffern im Lagebericht gefragt sowie die Ansichten der Entscheidungsträger, d.h. von Vorstand und Aufsichtsrat, zur Erhöhung des Frauenanteils in ihrem Unternehmen eingeholt.

Durch wiederholte Teilnahme an den Versammlungen und Stellen dieser Fragen sollte ein Rechtfertigungsdruck aufgebaut werden. So wird es möglich sein, Aussagen von Vorstand und Aufsichtsrat derselben Unternehmen an ihren Angaben vom Vorjahr zu messen. Von Januar bis Juli 2010 besuchten 72 Mitglieder des djb, der ARGE Anwältinnen sowie Interessentinnen anderer Professionen, darunter auch eine Journalistin, eine Ingenieurin und eine Psychologin, die Hauptversammlungen aller DAX30- und weiterer 44 Unternehmen aus dem TecDAX und MDAX. Alle Besuche erfolgten ehrenamtlich.

Ausgangspunkt des Projektes „Aktionärinnen fordern Gleichberechtigung“ ist die uneingeschränkte Entsprechenserklärung in Bezug auf Diversity, die von sämtlichen DAX30sowie den weiteren vom djb besuchten 44 TecDAX- und MDAX-Unternehmen abgegeben wurde. Alle vom djb besuchten Unternehmen sind börsennotiert und damit verpflichtet, eine Entsprechenserklärung nach $\mathbb{S} 161 \mathrm{AktG}$ abzugeben. Sie müssen erklären, inwieweit sie die Empfehlungen des Deutschen Corporate Governance Kodex (DCGK) vom 18. Juni 2009 zur Besetzung von Aufsichtsrat und Vorstand eingehalten haben oder von ihnen abweichen. Dabei ist unter Diversity nach Angaben der Regierungskommission auch die Berücksichtigung von Frauen zu verstehen. 